PROCEEDINGS OF THE

AMERICAN MATHEMATICAL SOCIETY

Volume 132, Number 9, Pages 2733-2742

S 0002-9939(04)07451-9

Article electronically published on March 25, 2004

\title{
UNCOUNTABLE CATEGORICITY FOR GROSS MODELS
}

\author{
MICHAEL C. LASKOWSKI AND ANAND PILLAY \\ (Communicated by Carl G. Jockusch, Jr.)
}

\begin{abstract}
A model $M$ is said to be gross if all infinite definable sets in $M$ have the same cardinality (as $M$ ). We prove that if for some uncountable $\kappa, T$ has a unique gross model of cardinality $\kappa$, then for any uncountable $\kappa, T$ has a unique gross model of cardinality $\kappa$.
\end{abstract}

\section{INTRODUCTION}

$T$ will denote a countable complete theory. Morley's theorem [6] states that if for some uncountable cardinal $\kappa, T$ has a unique model of cardinality $\kappa$, then for any uncountable cardinal $\kappa, T$ has a unique model of cardinality $\kappa$. Moreover, Baldwin and Lachlan [2] subsequently characterized these uncountably categorical theories as being precisely $\omega$-stable theories with no Vaughtian pairs. We do the same thing here, but restrict our attention to "gross models", where $M \models T$ is gross if any infinite definable set $X$ in $M$ has cardinality that of $M$. In Theorem 1.7 below we prove that $T$ has a unique gross model in some uncountable cardinality if and only if $T$ has a unique gross model in each uncountable cardinality. Also included is a characterization of such "uncountably gross-categorical theories": $T$ should be $\omega$-stable and any type over a model should be captured by types of CantorBendixon rank 1 in a precise sense described below. A stronger notion of grossness is obtained by working in $T^{e q}$. That is, define $M$ to be $e q$-gross if every infinite definable set in $M^{e q}$ has cardinality that of $M$. Morley's theorem also holds for $e q$ gross models, but the characterization of uncountably eq-gross-categorical theories is somewhat cleaner: $T$ is $\omega$-stable and any nonalgebraic type is nonorthogonal to a type of Morley rank 1. (See Theorem 3.4 and Remark 3.5.) In particular, we see (Corollary 3.6) that if $T$ is $\omega$-stable and $\omega$-categorical, then $T$ has a unique $e q$-gross model in every uncountable cardinality.

The topic of this paper arose from an examination of various sufficient conditions for saturation in Rahim Moosa's thesis [7]. In 4] Keisler investigates classes of structures that are $P C_{\delta}$ over $L_{\omega_{1}, \omega}$, which is a generalization of grossness. However, with the exception of our Lemma 2.1] none of our results are contained there. In our opinion, the most interesting results of the paper are the characterizations of

Received by the editors June 9, 2003.

2000 Mathematics Subject Classification. Primary 03C45; Secondary 03C50, 03C75.

The first author was partially supported by NSF grant DMS-0071746.

The second author was partially supported by NSF grants DMS-0070179 and DMS 01-00979 and a Humboldt Foundation Research Award. 
uncountably gross-categorical and eq-gross-categorical classes and the differences between them.

In the remainder of the introduction we give precise definitions, discuss the complexity of the class of gross models, and state our main theorem. Section 2 contains a number of lemmas that culminate in a proof of Theorem 1.7. In Section 3 we discuss the many-sorted case as well as eq-grossness.

Let us repeat that $T$ denotes a complete theory with infinite models in a countable language $L$. For now $T$ can be taken to be 1 -sorted. So the notion of the cardinality $M$ of a model of $T$ is unproblematic. Notation is fairly standard. Throughout "definable" always means "definable with parameters" and by a definable set in $M \models T$ we mean a subset of some $M^{n}$ definable in $M$. We typically work in a large, very saturated model $\mathfrak{C}$ of $T$. In Sections 1 and 2 it is important that we are working in the original language $L$ as opposed to $L^{e q}$. Consequently, the number of variables in a formula is relevant. A 1-type is a type in a single variable, usually denoted $x$. We denote the set of complete 1-types over a model by $S_{1}(M)$. By contrast, the term "type" refers to a (partial) $n$-type with parameters from $\mathfrak{C}$ for an unspecified $n$, and $S(M)$ denotes the union of the sets of complete $n$-types over $M$ for each $n$. In Section 3 we discuss the many-sorted case as well as $T^{e q}$.

Although the model theory used in this paper is quite straightforward, in Section 3 we use canonical bases in the context of a variant of Shelah's semiregular types theorem. In any case see [1], 11] and [8] for the required stability-theoretic background and results.

Definition 1.1. A structure $M$ is gross if every infinite definable subset of $M$ has size $|M|$.

A simple counting argument implies that $M$ is gross if and only if every infinite definable subset of $M^{1}$ has size $|M|$.

It is readily seen that the class of gross models of $T$ is $P C_{\delta}$ with omitting a type 1 For example, take $L^{\prime}$ to be the expansion of $L$ formed by adding a new unary predicate symbol $V$, new constant symbols $\left\{c_{n}: n \in \omega\right\}$, and, for each $L$ formula $\varphi(x, \bar{y})$, two new relation symbols $R_{\varphi}(\bar{y}, z)$ and $S_{\varphi}(x, \bar{y}, w)$. Take $T^{\prime}$ to be the $L^{\prime}$-theory containing $T$ with axioms ensuring that the $c_{n}$ 's are distinct, for every $n$ and $\varphi, V\left(c_{n}\right)$ holds, $\forall \bar{y}\left[R_{\varphi}\left(\bar{y}, c_{n}\right) \leftrightarrow \exists^{=n} x \varphi(x, \bar{y})\right]$, together with a single axiom stating that for every $\bar{y}$, either $\exists z\left(V(z) \wedge R_{\varphi}(\bar{y}, z)\right)$ or $S_{\varphi}(x, \bar{y}, w)$ describes a bijection between $M^{\prime}$ and $\varphi\left(M^{\prime}, \bar{y}\right)$ for any $M^{\prime} \models T^{\prime}$. Additionally, let $p(z)$ denote the type $\{V(z)\} \cup\left\{z \neq c_{n}: n \in \omega\right\}$. Then the class of gross models of $T$ is precisely the class of reducts of models of $T^{\prime}$ that omit $p$.

In general, the class of gross models is not a $P C_{\delta}$ class. To delineate when it is, recall that a theory $T$ eliminates infinity if for every $L$-formula $\varphi(x, \bar{y})$ there is an integer $N_{\varphi}$ such that for any model $M$ of $T$ and any $\bar{a}$ from $M$, either $\varphi(M, \bar{a})$ is infinite or has size at most $N_{\varphi}$.

Proposition 1.2. Let $T$ be any theory in a countable language. The class of gross models of $T$ is a $P C_{\delta}$-class if and only if $T$ eliminates infinity.

Proof. First, if $T$ eliminates infinity, then the construction of $T^{\prime}$ above can be modified by replacing $\exists z\left(V(z) \wedge R_{\varphi}(\bar{y}, z)\right)$ by $\bigvee_{n \leq N_{\varphi}} R_{\varphi}\left(\bar{y}, c_{n}\right)$ for every $L$-formula $\varphi$, which obviates the need for the type $p$ to be omitted.

${ }^{1}$ A straightforward inductive argument shows that a class $K$ of structures is $P C_{\delta}$ omitting a type if and only if $K$ is $P C_{\delta}$ over $L_{\omega_{1}, \omega}$ in the sense of Keisler [4]. 
Conversely, suppose that there is an $L$-formula $\varphi(x, \bar{y})$ witnessing that $T$ does not eliminate infinity. Then there is a countable model $M$ of $T$ and an infinite subset $A \subseteq \omega$ such that for every $n \in A$ there is $\bar{a}_{n}$ from $M$ with $\varphi\left(M, \bar{a}_{n}\right)$ having exactly $n$ elements. By way of contradiction suppose that the class of gross models of $T$ is $P C_{\delta}$, i.e., there is a countable $L^{\prime} \supseteq L$ and an $L^{\prime}$-theory $T^{\prime}$ such that the class of gross models of $T$ is precisely the class of $L$-reducts of models of $T^{\prime}$. Then clearly, since every countable structure is gross, $M$ is the reduct of some $M^{\prime} \models T^{\prime}$.

Now expand $M^{\prime}$ to $M^{\prime \prime}$ as follows: Let $s=l g(\bar{y})$. First, add a new $s$-ary relation symbol $P$ with interpretation $\left\{\bar{a}_{n}: n \in A\right\}$ and add a new $2 s$-ary relation $<$ on $P^{2}$ whose interpretation satisfies $\bar{a}_{n}<\bar{a}_{m}$ if and only if $n<m$. Next, Skolemize this expanded structure, obtaining a larger (countable) $L^{\prime \prime} \supseteq L^{\prime} \cup\{P,<\}$ and our expansion $M^{\prime \prime}$.

Note that by Ramsey's theorem, given any infinite subset $B \subseteq A$ and any $L^{\prime \prime}$ formula $\psi$, there is an infinite $B^{\prime} \subseteq B$ that is $\{\psi\}$-indiscernible. Thus, by compactness (as in the proof of the Ehrenfeucht-Mostowski theorem) there is a model $N^{\prime \prime}$ of $T^{\prime \prime}$ and a set of $s$-tuples $\left\{\bar{c}_{\alpha}: \alpha<\omega_{1}\right\}$ from $N^{\prime \prime}$ such that:

(1) $\left\langle\bar{c}_{\alpha}: \alpha<\omega_{1}\right\rangle$ are order-indiscernible;

(2) $N^{\prime \prime}$ is the Skolem Hull of $\left\{\bar{c}_{\alpha}: \alpha<\omega_{1}\right\}$;

(3) for any $\alpha_{1}<\cdots<\alpha_{n}<\omega_{1}$ and any $L^{\prime \prime}$-formula $\psi\left(\bar{x}_{1}, \ldots, \bar{x}_{n}\right)$, if $N^{\prime \prime} \models$ $\psi\left(\bar{c}_{\alpha_{1}}, \ldots, \bar{c}_{\alpha_{n}}\right)$ for some (equiv. for any) $\alpha_{1}<\cdots<\alpha_{n}<\omega_{1}$, then there is an infinite subset $B \subseteq A$ such that $M^{\prime \prime} \models \psi\left(\bar{a}_{j_{1}}, \ldots, \bar{a}_{j_{n}}\right)$ for all $j_{1}<\cdots<$ $j_{n}$ from $B$.

In particular, $N^{\prime \prime} \models P\left(\bar{c}_{\alpha}\right) \wedge\left(\bar{c}_{\alpha}<\bar{c}_{\beta}\right)$ for all $\alpha<\beta<\omega_{1}$. Clearly, $N^{\prime \prime}$ has size $\aleph_{1}$. Our desired contradiction follows from the following claim.

Claim. The set $\varphi\left(N^{\prime \prime}, \bar{c}_{\alpha}\right)$ is countably infinite for all $\alpha<\omega_{1}$.

Proof. Fix $\alpha^{*}<\omega_{1}$. First, for each $l \in \omega$, since only finitely many elements of $P\left(M^{\prime \prime}\right)=\left\{\bar{a}_{n}: n \in A\right\}$ satisfy $\exists^{<l} x \varphi\left(x, \bar{a}_{n}\right), \varphi\left(N^{\prime \prime}, \bar{c}_{\alpha^{*}}\right)$ has at least $l$ elements. Hence $\varphi\left(N^{\prime \prime}, \bar{c}_{\alpha^{*}}\right)$ is infinite.

By way of contradiction, suppose that $\varphi\left(N^{\prime \prime}, \bar{c}_{\alpha^{*}}\right)$ were uncountable. Since $L^{\prime \prime}$ is countable there would be an $L^{\prime \prime}$-term $\tau\left(\bar{x}_{1}, \ldots, \bar{x}_{n}\right)$ and uncountably many, strictly increasing $n$-tuples $\left\langle\bar{\alpha}_{j}: j \in \omega_{1}\right\rangle$ of elements from $\omega_{1}$ such that, letting $\hat{c}_{j}$ denote the $n s$-tuple $\left(\bar{c}_{\alpha_{j 1}}, \ldots, \bar{c}_{\alpha_{j n}}\right)$,

$$
N^{\prime \prime} \models \tau\left(\hat{c}_{j}\right) \neq \tau\left(\hat{c}_{l}\right) \wedge \varphi\left(\tau\left(\hat{c}_{j}\right), \bar{c}_{\alpha^{*}}\right)
$$

for all $j<l<\omega_{1}$. Without loss of generality, we may assume that for some $1 \leq k \leq n, \alpha^{*}$ is the $k^{\text {th }}$ element of $\bar{\alpha}_{j}$ for all $j<\omega_{1}$.

By the $\Delta$-system lemma (or more precisely by its proof) there is an $m, k \leq m<n$ and an uncountable $X \subseteq \omega_{1}$ such that for all $j<l<\omega_{1}$, the first $m$ entries of $\bar{\alpha}_{j}$ and $\bar{\alpha}_{l}$ are the same, and, letting $\bar{\beta}_{j}$ denote the subsequence of $\bar{\alpha}_{j}$ consisting of the final $n-m$ entries, every element of $\bar{\beta}_{j}$ is less than every element of $\bar{\beta}_{l}$. Choose any $j<l$ from $X$ and let $\psi$ be the formula described in (4). Then, translating to $M^{\prime \prime}$, let $b_{1}<\cdots<b_{m}$ denote the first $m$ elements of $B \subseteq A$, where $B$ is chosen to correspond to $\{\psi\}$ in Clause $(3)$ above. Then, any $(n-m)$-tuples of integers $d_{m+1}, \ldots, d_{n}$, $e_{m+1}, \ldots, e_{n}$ from $B$ satisfying $b_{m}<d_{m+1}<\cdots<d_{n}<e_{m+1}<\cdots<e_{n}$ will necessarily satisfy

$$
M^{\prime \prime} \models \tau\left(\bar{a}_{b_{1}}, \ldots, \bar{a}_{b_{m}}, \bar{a}_{d_{m+1}}, \ldots, \bar{a}_{d_{n}}\right) \neq \tau\left(\bar{a}_{b_{1}}, \ldots, \bar{a}_{b_{m}}, \bar{a}_{e_{m+1}}, \ldots, \bar{a}_{e_{n}}\right)
$$


as well as $M^{\prime \prime} \models \varphi\left(\tau\left(\bar{a}_{b_{1}}, \ldots, \bar{a}_{b_{m}}, \bar{a}_{d_{m+1}}, \ldots, \bar{a}_{d_{n}}\right), \bar{a}_{b_{k}}\right)$. Hence, $\varphi\left(M^{\prime \prime}, \bar{a}_{b_{k}}\right)$ is infinite, a contradiction.

Example 1.8(c) gives a theory $T$ that does not eliminate infinity, yet has exactly one gross model in every uncountable cardinality. Despite the complexity of describing the class of gross models, such classes do have nice closure properties. Each of the following observations is easily verified:

Remark 1.3. (i) Any countable model of $T$ is gross.

(ii) For every model $M$ of $T$ and every cardinal $\kappa \geq|M|$, there is a gross elementary extension of $M$ of size $\kappa$.

(iii) The union of a countable elementary chain of gross models of $T$ is gross.

The central notion in the analysis of gross models is that of a type over a model having Cantor-Bendixon (CB) rank 1. Recall:

Definition 1.4. A 1-type $p \in S_{1}(M)$ has $C B$-rank 1 if $p$ is nonalgebraic and there is a formula $\varphi(x) \in p$ such that for any $L(M)$-formula $\psi(x, \bar{c})$, one of $\varphi(x) \wedge \psi(x, \bar{c})$ or $\varphi(x) \wedge \neg \psi(x, \bar{c})$ is algebraic. An $L(M)$-formula $\varphi(x)$ has $C B$-Rank 1, Multiplicity 1 if there is a unique $p \in S_{1}(M)$ of CB-rank 1 with $\varphi \in p$.

When $M$ is $\omega$-saturated, then $M$ has type CB-rank 1 if and only if it has Morley rank 1 , but $C B$-rank 1 types occur over nonsaturated models as well. In particular, if $M$ is any model of an $\omega$-stable theory, then every nonalgebraic formula over $M$ with one free variable is an element of a CB-rank 1 type. A CB-rank 1 type is clearly strongly regular, but a strongly regular type in $S_{1}(M)$ need not have CB-rank 1 over $M$. As an example, let $T$ be the theory of a single equivalence relation $E$ with infinitely many classes, each infinite. Then for any $M \models T$, the type $p(x) \in S_{1}(M)$ asserting that $x$ is in a new $E$-class is strongly regular but not of CB-rank 1 .

Definition 1.5. A $C B$-rank 1 construction over $M$ is a sequence $\left\langle\left(a_{i}, M_{i}\right): i \in \alpha\right\rangle$ such that

(1) $M_{0}=M$;

(2) $M_{i+1}$ is prime over $M_{i} a_{i}$;

(3) $\operatorname{tp}\left(a_{i} / M_{i}\right)$ has CB-rank 1 ; and

(4) $M_{\beta}=\bigcup\left\{M_{i}: i<\beta\right\}$ for all nonzero limit ordinals $\beta<\alpha$.

Definition 1.6. A type $p \in S(M)$ is accessible if there is a CB-rank 1 construction $\left\langle\left(a_{i}, M_{i}\right): i \in \alpha\right\rangle$ over $M$ such that $M_{\alpha}$ realizes $p$.

Theorem 1.7. The following are equivalent for any complete theory $T$ with infinite models in a countable language:

(1) for some uncountable cardinal $\kappa, T$ has exactly one gross model of size $\kappa$;

(2) $T$ is $\omega$-stable and every type over every countable model of $T$ is accessible;

(3) $T$ is $\omega$-stable and every type over every model of $T$ is accessible;

(4) every uncountable gross model is saturated;

(5) for every uncountable cardinal $\kappa, T$ has exactly one gross model of size $\kappa$.

Example 1.8. (a) There are ( $\omega$-categorical) theories satisfying Conditions (1)-(5) in the theorem above that have the DOP, and so have $2^{\kappa}$ models of cardinality $\kappa$ for all $\kappa>\omega$.

(b) There are theories satisfying Conditions (1)-(5) in the theorem above that have infinite Morley rank. 
(c) There are theories satisfying Conditions (1)-(5) in the theorem above that do not eliminate infinity.

(a): The basic example of an $\omega$-categorical, $\omega$-stable theory with the DOP works. This is the theory of a structure with an infinite set of "vertices" and between any two vertices there is an infinite set of "edges".

(b): Consider a structure with three predicates $P, Q$ and $V$, an equivalence relation $E$ on $Q$, a unary function $g$, and $n$-ary functions $f_{n}$ for $1 \leq n<\omega$. $P$ is infinite, and for each $n, f_{n}$ is a bijection between $P^{n}$ and an $E$-equivalence class $U_{n}$ on $Q$ (where $U_{n}$ and $U_{m}$ are distinct if $n \neq m$ ). All $E$-classes on $Q$ are infinite. As well, $g: Q \rightarrow V$ is the canonical surjection induced by $E$ (i.e., $g(a)=g(b)$ iff $E(a, b))$. Note that $R M\left(U_{n}\right)=n$ for all $n$ whereby $R M(Q)=\omega$. On the other hand, any $E$-class different from each $U_{n}$ has CB-rank 1 and CB-multiplicity 1 , as have the formulas $P(x)$ and $V(x)$.

(c): Take disjoint, unary predicates $Q, V$ and an equivalence relation $E$ on $Q$. For each finite $n>0, Q$ contains exactly one $E$-class with exactly $n$ elements. As in (b) the language includes the canonical surjection $g: Q \rightarrow V$ induced by $E$.

\section{Proof of Theorem 1.7}

We begin by showing that uniqueness of gross models of some uncountable cardinality implies $\omega$-stability of $T$. The crucial point is the following lemma, which gives gross models realizing few types over countable sets. If $T$ eliminates infinity, this would be a trivial Ehrenfeucht-Mostowski argument. But in general we must do a bit more. This fact was also proved by Keisler (see Theorem 33 of [4]), and Shelah gives a sketch of a proof in Step 1 of the proof of Theorem 5.2 in [9].

Lemma 2.1 ( $T$ any countable complete theory). For any uncountable cardinal $\kappa$, $T$ has a gross model $M$ of cardinality $\kappa$ such that for any countable subset $A$ of $M$, only countably many complete 1-types over $A$ are realized in $M$.

Proof. Let $N$ be a gross model of $T$ of cardinality $\lambda \geq \beth_{\left(2^{|T|}\right)^{+}}$. Enlarge the language $L$ of $T$ to a language $L^{\prime}$ by adjoining a predicate symbol $P_{\varphi}(\bar{y})$ and function symbol $f_{\varphi}(\bar{y}, x)$ for each formula $\varphi(x, \bar{y}) \in L$. Expand $N$ to an $L^{\prime}$-structure $N^{\prime}$, by interpreting $P_{\varphi}$ as the set of $\bar{c}$ from $N$ such that $\varphi(x, \bar{c})$ is algebraic (has finitely many realizations), and by interpreting $f_{\varphi}(\bar{c},-)$ as a bijection between $\varphi(x, \bar{c})^{N}$ and $N$ if $\neg P_{\varphi}(\bar{c})$ and anything you want otherwise. Note that $L^{\prime}$ is countable. Now add Skolem functions to $N^{\prime}$ to get an expansion $N^{\prime \prime}$, in a countable language $L^{\prime \prime}$ with theory $T^{\prime \prime}$ say.

Fix $\kappa>\omega$. Using Erdös-Rado (see [10] or Lemma A.3 of [5]) we can find an $\left(L^{\prime \prime}\right)$-indiscernible sequence $\left\langle a_{i}: i<\kappa\right\rangle$ in some model of $T^{\prime \prime}$, such that for every $n, t p_{L^{\prime \prime}}\left(a_{0}, \ldots, a_{n-1}\right)$ is realized in $N^{\prime \prime}$. Let $M^{\prime \prime}$ be the Skolem hull of $\left\{a_{i}: i<\kappa\right\}$, and $M$ the $L$-reduct of $M^{\prime \prime}$. So $M$ is a model of $T$ of cardinality $\kappa$ that realizes only countably many types over any countable subset.

It remains to prove that $M$ is gross. Now $M^{\prime \prime} \mid L^{\prime}$ is a model of $T h\left(N^{\prime}\right)$. So whenever $\bar{c}$ is from $M$, and $\neg P_{\varphi}(\bar{c})$ holds in $M^{\prime \prime}, \varphi(x, \bar{c})^{M}$ has cardinality $\kappa$. On the other hand, every (partial) finitary type of $T^{\prime \prime}$ realized in $M^{\prime \prime}$ must be realized in $N^{\prime \prime}$. But for any $\varphi(x, \bar{y}) \in L, N^{\prime \prime}$ omits the type: $\left\{P_{\varphi}(\bar{y})\right\} \cup\{$ there are infinitely many realizations of $\varphi(x, \bar{y})\}$. So $M^{\prime \prime}$ omits this type too. What this means is that if $\bar{c}$ is from $M$ and $\varphi(x, \bar{c})^{M}$ is infinite, then $M^{\prime \prime} \models \neg P_{\varphi}(\bar{c})$; so as mentioned above $\varphi(x, \bar{c})^{M}$ has size $\kappa$. 
Corollary 2.2. Suppose $\kappa$ is uncountable and that $T$ has a unique gross model of cardinality $\kappa$ (up to isomorphism). Then $T$ is $\omega$-stable.

Proof. If not, there is a countable model $M_{0}$ of $T$ over which there are uncountably many types. Choose a model $M$ of cardinality $\kappa$ containing $M_{0}$ and realizing uncountably many of these types. It is easy to extend $M$ to a gross model of cardinality $\kappa$. Then $M$ is not isomorphic to the gross model of cardinality $\kappa$ given by Lemma 2.1 .

The following two lemmas, which describe the relationship between CB-rank 1 types and gross models, rely on the ubiquity of types of CB-rank 1 in $S_{1}(M)$ when $M$ is a model of an $\omega$-stable theory.

Lemma 2.3. Suppose that $T$ is $\omega$-stable. For every countable model $M$ of $T$ and every uncountable $\kappa$, there is a CB-rank 1 construction of a gross model $N$ extending $M$ of size $\kappa$.

Proof. This is essentially bookkeeping. The point is that if $\left\langle\left(a_{i}, M_{i}\right): i<\alpha\right\rangle$ is a CB-rank 1 construction over $M$, then by choosing $M_{\alpha}$ to be prime over $M_{\alpha-1} a_{\alpha-1}$ when $\alpha$ is a successor ordinal, and equal to $\bigcup\left\{M_{\beta}: \beta<\alpha\right\}$ when $\alpha$ is a limit, then for every nonalgebraic formula $\varphi(x, \bar{c})$ over $M_{\alpha}$ one can find a realization $a_{\alpha}$ of $\varphi(x, \bar{c})$ such that $\operatorname{tp}\left(a_{\alpha} / M_{\alpha}\right)$ is CB-rank 1. Thus, $\left\langle\left(a_{i}, M_{i}\right): i \leq \alpha\right\rangle$ is a CB-rank 1 construction over $M$ and we can continue.

Lemma 2.4. Suppose that $T$ is $\omega$-stable. If $\left\langle\left(a_{i}, M_{i}\right): i \in \alpha\right\rangle$ is a CB-rank 1 construction over $M$ and $\kappa>|M|+|\alpha|$, then for any gross model $N$ extending $M$ of size $\kappa$ there is an elementary $f: M_{\alpha} \rightarrow N$ extending the identity map on $M$.

Proof. Arguing by induction on $i<\alpha$, it suffices to show that whenever $N$ is a gross extension of $M,|N|>|M|$, and $p=\operatorname{tp}(a / M)$ has CB-rank 1, then $p$ is realized in $N$. Let $\varphi(x) \in p$ witness that $p$ has $C B$-rank 1 (and multiplicity 1 ). $\varphi(x)$ has $|N|$-many realizations in $N$, so is realized by some $a \in N \backslash M$. But then $a \notin \operatorname{acl}(M)$; so $a$ realizes $p$.

An obstruction that one faces when working with CB-rank 1 types over a model is that CB-rank is typically not preserved under nonforking extensions. However, if the base model is relatively $\omega$-saturated in the extension, then this obstruction is eliminated. Moreover, the assumption of relative $\omega$-saturation can be preserved along CB-rank 1 constructions. These preservation results are summarized in the following lemma. Recall that if $M$ is an elementary substructure of $N$, we say that $M$ is relatively $\omega$-saturated in $N$ if every type over a finite subset of $M$ that is realized in $N$ is realized in $M$.

Lemma 2.5. Suppose that $T$ is $\omega$-stable and $M$ is a relatively $\omega$-saturated submodel of a model $N$ of $T$.

(1) If $q \in S(N)$ is the nonforking extension of a type $p \in S(M)$, then $p$ has $C B$-rank 1 if and only if $q$ has CB-rank 1.

(2) If $\operatorname{tp}(a / N)$ does not fork over $M, N(a)$ is prime over $N a$, and $M(a) \preceq N(a)$ is prime over $M a$, then $M(a)$ is relatively $\omega$-saturated in $N(a)$.

Proof. (1) Since being nonalgebraic is absolute, $q$ has CB-rank 1 implies $p$ has CB-rank 1 whenever $M \preceq N$. Conversely, suppose that $q$ does not have CB-rank 1 and choose any $\varphi(x, \bar{a}) \in p$. Then there is an $L(N)$-formula $\psi(x, \bar{c})$ such that 
both $\varphi(x, \bar{a}) \wedge \psi(x, \bar{c})$ and $\varphi(x, \bar{a}) \wedge \neg \psi(x, \bar{c})$ are infinite. Choose $\bar{c}^{\prime}$ from $M$ such that $\operatorname{tp}\left(\bar{c}^{\prime} / a\right)=\operatorname{tp}(\bar{c} / a)$. Then the $L(M)$-formula $\psi\left(x, \bar{c}^{\prime}\right)$ demonstrates that the CB-rank-multiplicity (over $M$ ) of $\varphi(x, \bar{a})$ is not $(1,1)$.

(2) Fix a finite tuple $\bar{b}$ from $M(a)$ and choose $\bar{c}$ from $M$ such that $\operatorname{tp}(\bar{b} / M a)$ is isolated by a formula over $a \bar{c}$. Choose $d \in N(a)$ arbitrarily. We will show that $\operatorname{tp}(d / \bar{b})$ is realized in $M(a)$. To this end, $\operatorname{suppose} \operatorname{tp}(d / N a)$ is isolated by $\theta(x, a, \bar{e})$. Choose $\bar{e}^{\prime}$ from $M$ with $\operatorname{tp}\left(\bar{e}^{\prime} / \bar{c}\right)=\operatorname{tp}(\bar{e} / \bar{c})$. It is readily checked that $\theta\left(x, a, \bar{e}^{\prime}\right)$ isolates $\operatorname{tp}(d / \bar{b})$; hence this type is realized in $M(a)$.

Lemma 2.6. If $p \in S(M)$ is accessible, then $p$ is accessible via a CB-rank 1 construction of finite length.

Proof. Suppose that $\left\langle\left(a_{i}, M_{i}\right): i \in \alpha\right\rangle$ is a CB-rank 1 construction over $M$ such that $M_{\alpha}$ realizes $p$. We set some notation:

- For any $c \in M_{\alpha}, r(c)=$ the least $\beta$ such that $c \in M_{\beta}$.

Note that the ordinal $r(c)$ is a successor as long as it is positive. So let $\rho(c)=r(c)-1$ for every $c \in M_{\alpha} \backslash M$. (In particular $\rho\left(a_{i}\right)=i$.)

- For a finite $F \subseteq M_{\alpha}$, let the closure of $F$ be $F \cup\left\{a_{\rho(c)}: c \in F\right\}$ and let $r(F)=\max \{r(c): c \in F\}$.

Note that the closure of a finite set is finite, $r(F)=r($ closure of $F)$, and $r(F)=0$ if and only if $F \subseteq M$.

- For each $i \in \alpha$, choose $D_{i} \subseteq M_{i}$ finite such that there is a formula $\varphi\left(x, D_{i}\right) \in$ $\operatorname{tp}\left(a_{i} / M_{i}\right)$ of CB-rank-multiplicity $(1,1)$ over $M_{i}$.

- For each $c \in M_{\alpha}$, choose $B(c) \subseteq M_{\rho(c)}$ finite such that $\operatorname{tp}\left(c / a_{\rho(c)} B(c)\right)$ isolates $\operatorname{tp}\left(c / a_{\rho(c)} M_{\rho(c)}\right)$.

- For $F \subseteq M_{\alpha}$, let $F^{\prime}=\bigcup\left\{D_{i} \backslash M: a_{i} \in F\right\} \cup \bigcup\{B(c) \backslash M: c \in F \backslash M\}$.

Note that $r\left(F^{\prime}\right)<r(F)$ as long as $r(F)>0$.

Now choose $\bar{c}$ from $M_{\alpha}$ realizing $p$. Let $F_{0}$ denote the closure of $\bar{c}$. Given a finite, closed $F_{n}$, let $F_{n+1}$ be the closure of $F_{n}^{\prime}$. Since $\alpha \geq r\left(F_{0}\right)>r\left(F_{1}\right)>\cdots$ whenever the sets are nonempty, it follows that there is $n$ such that $F_{n}=\emptyset$. Let $G=\bigcup\left\{F_{l}: l<n\right\}$, and let $H=\{r(c): c \in G\}$. It is easily checked that $\left\langle\left(a_{i}, M_{i}^{\prime}\right): i \in H\right\rangle$, where $M_{0}^{\prime}=M$ and the "next" $M^{\prime}$ is prime over $M_{i}^{\prime} a_{i}$, is a CB-rank 1 construction over $M$ that realizes $p$.

Lemma 2.7. Suppose that $T$ is $\omega$-stable and every type $p \in S(M)$ is accessible for every countable model $M$ of $T$. Then every type over every model of $T$ is accessible.

Proof. Let $N$ be any model of $T$ and choose $q \in S(N)$ arbitrarily. Choose $B \subseteq N$ finite such that $q$ is based and stationary over $B$. Take $M \supseteq B$ to be any countable, relatively $\omega$-saturated substructure of $N$, and let $p$ denote the restriction of $q$ to $M$. Let $\left\langle\left(a_{i}, M_{i}\right): i \in k\right\rangle$ be a CB-rank 1 construction over $M$ such that $M_{k}$ realizes $p$ (by Lemma 2.6 we may assume $k<\omega$ ). Without loss of generality, we may assume that $M_{k}$ is independent of $N$ over $M$. Construct any sequence $\left\langle N_{i}: i \in k\right\rangle$ satisfying $N_{0}=N, M_{i} \preceq N_{i}$, and $N_{i+1}$ is prime over $N_{i} a_{i}$ for each $i$. We claim that $\left\langle\left(a_{i}, N_{i}\right): i \in k\right\rangle$ is a CB-rank 1 construction over $N$. To see this, we argue that for each $i<k, M_{i}$ is relatively $\omega$-saturated in $N_{i}$ and $\operatorname{tp}\left(a_{i} / N_{i}\right)$ has CB-rank 1. But these statements are exactly the content of Lemma 2.5. which is applicable since $\operatorname{tp}\left(a_{i} / N\right)$ does not fork over $M_{i}$. Finally, it is clear that $N_{k}$ realizes $q$, since $M_{k}$ realizes $p$ and $M_{k}$ is independent of $N$ over $M$. 
Proof of Theorem 1.7. (1) $\Rightarrow(2)$. Choose an uncountable cardinal $\kappa$ such that $T$ has exactly one gross model $N$ of size $\kappa . T$ is $\omega$-stable because of Corollary 2.2 Thus, $N$ is saturated. Fix a countable model $M$ of $T$ and a type $p \in S(M)$. Since $N$ is saturated, we may assume $M \preceq N$. By Lemma 2.3 we may assume that $N$ is constructible over $M$ by a CB-rank 1 construction. However, since $N$ is saturated, it must surely realize $p$, and so $p$ is accessible.

$(2) \Rightarrow(3)$ is exactly Lemma 2.7

$(3) \Rightarrow(4)$. Fix an uncountable gross model $N$ of $T$. We argue that $N$ is saturated. Choose any $M \preceq N$ such that $|M|<|N|$ and choose any $p \in S(M)$. By (3) and Lemma 2.6 there is a CB-rank 1 construction $\left\langle\left(a_{i}, M_{i}\right): i<k\right\rangle$ over $M$ such that $M_{k}$ realizes $p$. Thus, by Lemma 2.4, $N$ realizes $p$ as well.

$(4) \Rightarrow(5)$ and $(5) \Rightarrow(1)$ are immediate.

\section{EXTENSIONS TO MANY-SORTED THEORIES AND $T^{e q}$}

Suppose now that $T$ is a many-sorted theory, still in a countable language (so in particular with at most countably many sorts). By the cardinality of $M \models T$ we mean the sum of the cardinalities of the $S^{M}$ for $S$ a sort. It is then natural to call $M \models T$ gross if every infinite definable subset of $S^{M}$ has cardinality $|M|$ for each sort $S$.

There is no problem in adapting the proofs from Section 2 to this context. What is more interesting is identifying what happens when we pass from $T$ to $T^{e q}$.

Definition 3.1. A model $M$ of $T$ is eq-gross if $M^{e q}$ is gross.

It is readily checked that $M$ is eq-gross if and only if for every definable $D \subseteq M^{n}$ and every definable equivalence relation $E$ on $M^{2 n}, D / E$ is either finite or has size $|M|$. The following example illustrates the distinction between the two versions of grossness, even in the context of $\omega$-categorical theories.

Example 3.2. Let $T$ be the ( $\omega$-categorical) theory of a single equivalence relation $E$ with infinitely many classes, each of which is infinite. Then for every uncountable $\kappa$, the model $M_{\kappa} \models T$ consisting of countably many $E$-classes, each of size $\kappa$, is gross but not eq-gross. Furthermore, for any uncountable cardinal $\kappa, T$ has exactly one eq-gross model of size $\kappa$, while it has nonisomorphic gross models of size $\kappa$.

Since $T$ being eq-gross is equivalent to $T^{e q}$ being gross, Theorem 1.7 provides us with a cheap proof of Morley's theorem for eq-gross models of a theory. However, we are able to provide a cleaner characterization of those theories having exactly one eq-gross model in some (every) uncountable power. The following lemma, which we state in greater generality than is needed here, is the primary reason why this notion is better behaved than grossness.

Lemma 3.3. Suppose that $T$ is a stable theory and $T=T^{e q}$. If $p \in S(A)$ is regular and nonorthogonal to some type of Morley rank 1 , then there is a type $q \in S(A)$ of Morley rank 1 that is not weakly orthogonal to $p$.

Proof. (This is as in the standard proof of the existence of semiregular types.) Let $M \supseteq A$ be chosen such that there are $a$ realizing $p \mid M$ and $b$ such that $t p(b / M)$ has Morley rank 1 and $a$ and $b$ fork over $M$ (so $b \in \operatorname{acl}(M a)$ ). Thus $a$ forks with $b M$ over $A$. Let $c \in C b(\operatorname{stp}(b M / a A)) \backslash \operatorname{acl}(A)$. Then $c \in \operatorname{acl}(a A) \cap d c l\left(b_{1} M_{1}, \ldots, b_{n} M_{n}\right)$, where $\left(b_{i} M_{i}\right)_{i}$ is a Morley sequence in $\operatorname{stp}(b M / a A)$. Then $\operatorname{tp}(c / A)$ is regular, and 
$c$ is independent of $M_{1} \cup \cdots \cup M_{n}$ over $A$. So $t p\left(c / M_{1} \cdots M_{n}\right)$ is regular. But $c$ is in the definable closure of some elements which have Morley rank 0 or 1 over $M_{1} \cup \cdots \cup M_{n}$. This all implies that $t p\left(c / M_{1} \cdots M_{n}\right)$ has Morley rank 1 and hence tp $(c / A)$ has Morley rank 1.

Theorem 3.4. Let $T$ be any complete theory in a countable language with infinite models. Then the following conditions are equivalent:

(1) $T$ has exactly one eq-gross model in some uncountable $\kappa$;

(2) $T$ is $\omega$-stable and every nonalgebraic eq-type is nonorthogonal to an eq-type of Morley rank 1;

(3) $T$ is $\omega$-stable and for every $M \models T$, every nonalgebraic $p \in S\left(M^{e q}\right)$ is nonweakly orthogonal to a type $q \in S\left(M^{e q}\right)$ of Morley rank 1 ;

(4) every uncountable eq-gross model is saturated;

(5) Thas exactly one eq-gross model in every uncountable $\kappa$.

Proof. Throughout this proof we work in $T^{e q}$. In fact, to ease notation, we assume $T=T^{e q}$. So when we write "type" or "model" we mean an eq-type or $M^{e q}$ with respect to the original theory.

$(1) \Rightarrow(2)$. This is like $(1) \Rightarrow(2)$ in the proof of Theorem 1.7, but within the category of $\omega$-saturated models of $T$. Suppose that $N$ is the only gross model of $T$ of size $\kappa>\omega$. Then $T$ is $\omega$-stable by Corollary 2.2 hence $N$ is saturated. In particular, the countable, saturated model $M$ embeds into $N$. Now if some nonalgebraic type were orthogonal to all types of Morley rank 1 , then some nonalgebraic type $p \in$ $S(M)$ would be orthogonal to all types of Morley rank 1. Now define a construction sequence $\left\langle\left(a_{i}, M_{i}\right): i<\alpha\right\rangle$ akin to a CB-rank 1 construction, but where each $M_{i+1}$ is chosen to be $\omega$-saturated and dominated by $a_{i}$ over $M_{i}$. Note that since each $M_{i}$ is $\omega$-saturated, a type $q \in S\left(M_{i}\right)$ is of CB-rank 1 if and only if it has Morley rank 1. Consequently, since $p$ was chosen to be orthogonal to all types of Morley rank 1 , one proves by induction on $i<\alpha$ that $\operatorname{tp}\left(a / M_{i}\right)$ does not fork over $M$ for any $a$ realizing $p$. In particular, $p$ is omitted in $M_{\alpha}$. However, since $M$ is itself $\omega$-saturated, it is routine to show that a gross model $N^{\prime}$ extending $M$ of size $\kappa$ is constructible by such a sequence. But, since there is only one gross model of size $\kappa$, $N^{\prime}$ is saturated; hence $p$ is realized in $N^{\prime}$. Thus, $p$ is nonorthogonal to $\operatorname{tp}\left(a_{i} / M_{i}\right)$ for some $i$, which is a contradiction.

$(2) \Rightarrow(3)$. This is exactly Lemma 3.3 (For the $\omega$-categorical case recall that every model is $\omega$-saturated.)

$(3) \Rightarrow(4)$. We claim that every type $p \in S(M)$ over every countable model $M$ is accessible. If this were not the case, then choose $M$ and $p \in S(M)$ not accessible with the Lascar rank $U(p)$ as small as possible. By (3) $p$ is weakly orthogonal to some $q \in S(M)$ of Morley rank 1. Choose $a$ realizing $q$ and let $M^{\prime}$ be prime over $M a$. Let $b$ be a realization of $p$ that forks with $a$ over $M$. Since $p^{\prime}=\operatorname{tp}\left(b / M^{\prime}\right)$ is a forking extension of $p$, there is a CB-rank 1 construction sequence over $M^{\prime}$ that realizes $p^{\prime}$. But then, concatenating $(a, M)$ to that construction sequence yields a CB-rank 1 construction over $M$ that realizes $p$; hence $p$ is accessible. Now since $T$ is $\omega$-stable and every type over a countable set is accessible, (4) follows from Theorem 1.7 .

$(4) \Rightarrow(5)$ and $(5) \Rightarrow(1)$ are immediate.

Remark 3.5. It is easily shown that Condition (3) of Theorem [3.4 is equivalent to " $T$ is $\omega$-stable and in every proper elementary pair $M \preceq N$ of models, $\varphi^{N} \backslash \varphi^{M} \neq \emptyset$ 
for some $L(M)$-formula of CB-rank 1, Multiplicity 1." This should be compared with " $T$ is uncountably categorical if and only if $T$ is $\omega$-stable and in every proper elementary pair $M \preceq N$ of models, $\varphi^{N} \backslash \varphi^{M} \neq \emptyset$ for every $L(M)$-formula of CBrank 1, multiplicity 1." (That the latter condition implies "no Vaughtian pairs" is immediate from the abundance of CB-rank 1 types over models.)

Corollary 3.6. If $T$ is $\omega$-stable and $\omega$-categorical, then every uncountable gross model of $T^{e q}$ is saturated.

Proof. By Theorem 3.4 it suffices to show that any nonalgebraic type is nonorthogonal to a type of Morley rank 1 (in $T^{e q}$ ). However, by [3], $T$ has finite Morley rank and thus every type has finite $U$-rank. Thus every nonalgebraic type is nonorthogonal to an $e q$-type of $U$-rank 1 . By $\omega$-categoricity (and superstability) any type of $U$-rank 1 has Morley rank 1.

Note that Example 3.2 indicates the necessity of passing to $T^{e q}$ for this result. Finally, the example of countably many disjoint, infinite unary predicates demonstrates that the result above cannot be generalized to $\omega$-stable theories with nfcp or of finite Morley rank.

\section{REFERENCES}

[1] J. T. Baldwin, Fundamentals of Stability Theory, Perspectives in Mathematical Logic, Springer-Verlag, Berlin, 1988. MR 89k:03002

[2] J. T. Baldwin and A. H. Lachlan, On strongly minimal sets, Journal of Symbolic Logic 36 (1971), 79-96. MR 44:3851

[3] G. Cherlin, L. A. Harrington, and A. H. Lachlan, $\aleph_{0}$-categorical, $\aleph_{0}$-stable structures, Annals of Pure and Applied Logic 28 (1985), 103-135. MR 86g:03054

[4] H. J. Keisler, Model theory for infinitary logic. Logic with countable conjunctions and finite quantifiers, North-Holland, Amsterdan, 1971. MR 49:8855

[5] B. Kim, Simple first order theories, Ph.D. thesis, University of Notre Dame, 1996.

[6] M. Morley, Categoricity in power, Trans. Amer. Math. Soc. 114 (1965), 514-538. MR 31:58

[7] R. Moosa, Contributions to the model theory of fields and compact complex spaces, Ph.D. thesis, University of Illinois at Urbana-Champaign, 2001.

[8] A. Pillay, Geometric Stability Theory, The Clarendon Press, Oxford University Press, New York, 1996. MR 98a:03049

[9] S. Shelah, The lazy model-theoretician's guide to stability, Logique et Analyse (N.S.) 18 (1975). MR 58:27447

[10] S. Shelah, Simple unstable theories, Annals of Mathematical Logic 19 (1980), 177-203. MR 82g:03055

[11] S. Shelah, Classification Theory and the Number of Nonisomorphic Models, 2nd ed., NorthHolland, Amsterdam, 1990. MR 91k:03085

Department of Mathematics, University of Maryland, College Park, Maryland 20742

E-mail address: mcl@math.umd.edu

Department of Mathematics, University of Illinois at Urbana-Champaign, Urbana, ILLINOIS 61801

E-mail address: pillay@math.uiuc.edu 